4 Yde J, Nielsen, AB. Sports injuires in adolescents' ball games: soccer, handball and basketball. $\mathrm{Br} \mathcal{F}$ Sports $\mathrm{Med}$ 1990;24:51-4.

5 Kirk, AA. Dunk lacerations: unusual injuries to the hands of basketball players. $¥ A M A$ 1979;242:415.

6 Urbaniak JR, Evans JP, Bright, DS. Microvascular management of ring avulsion injuries. $\mathcal{F}$ Hand Surg 1981;6:25-30. 7 Nissenbaum, M. Class IIA ring avulsion injuries: an absolute indication for microvascular repair. $\mathcal{f}$ Hand Surg 1984;9A:810-15.
8 Fahmy NRM, Sanderson PL, Barrie JL. A new technique in the management of the ring avulsion injury. Injury 1991;22:121-3.

9 DeBenedette V. Bad breaks in basketball: unforgettable injuries. Physics and Sports Medicine 1991;19:135-6.

10 Ellison LF. Basketball injuries in the database of the Canandian hospitals injury reporting and preventive program (CHIRPP). Chronic Disease in Canada 1995;16:117-24.

\title{
Bilateral locked posterior shoulder dislocation in a footballer
}

\author{
J Ryan, $M$ Whitten
}

\begin{abstract}
Posterior dislocation of the shoulder is an uncommon injury, accounting for between $2 \%$ and $4 \%$ of all shoulder dislocations. It occurs most frequently in patients following convulsions or direct anterior force to the shoulder. It is a particularly uncommon injury in sport. This paper reports an unusual case of bilateral locked posteriorly dislocated shoulders in a previously healthy young man who fell while playing football.

(Br F Sports Med 1997;31:74-75)
\end{abstract}

Keywords: posterior dislocation; shoulder; football.

\section{Case report}

A 26 year old man presented to the accident and emergency (A\&E) department with bilateral shoulder pain. He said that he had fallen backwards onto his right shoulder while

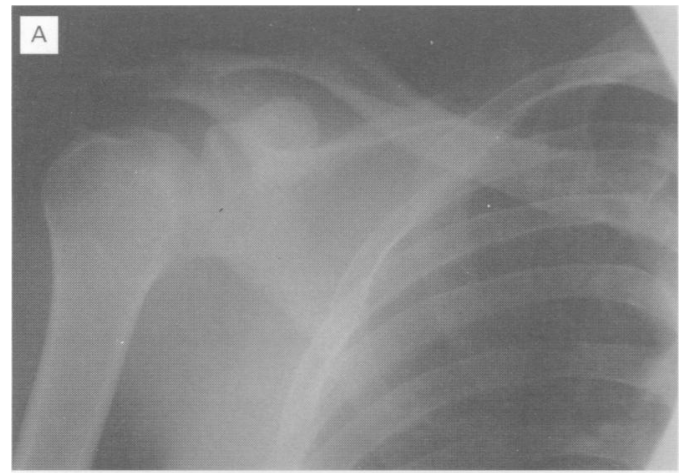

\section{Accident and}

Emergency

Department, King's

College Hospital,

Denmark Hill, London

SE5 9RS, United

Kingdom

J Ryan, senior registrar

$M$ Whitten, senior house officer

Correspondence to: Mr John Ryan, Department of Accident and Emergency Medicine, Royal Sussex

County Hospital, Brighton

BN2 1HG, United Kingdom.

Accepted for publication 29 April 1996

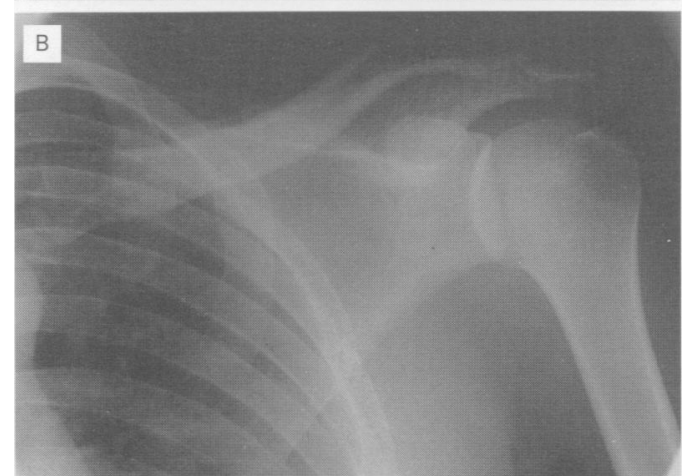

Figure 1 Anteroposterior radiographs of right and left shoulders.
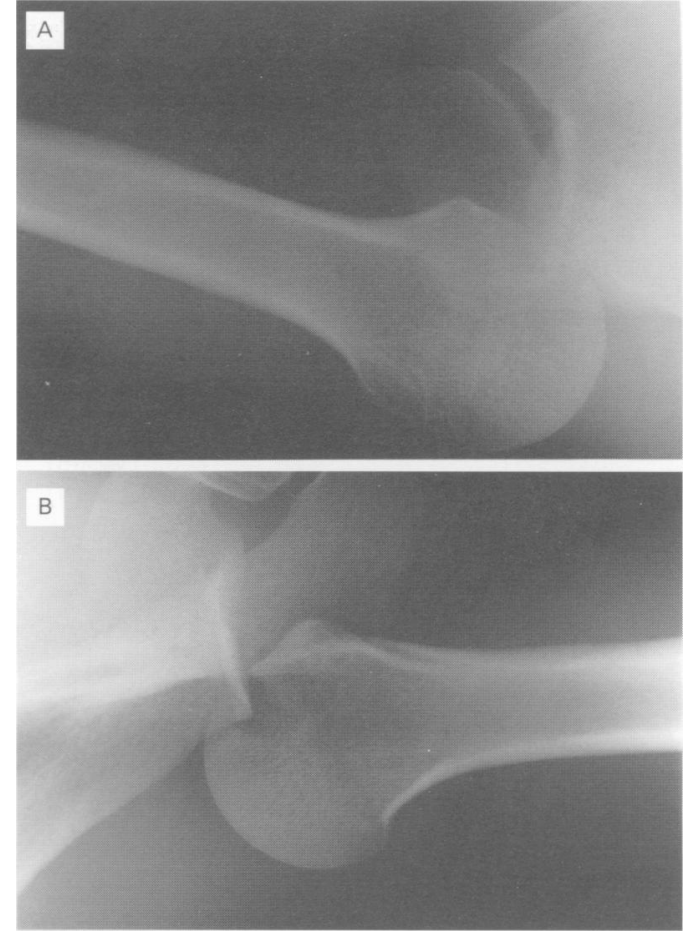

Figure 2 Axial view radiographs of right and left shoulders.

playing five-a-side soccer on hard ground. When he hit the ground he rolled onto his left side experiencing sudden pain in his left shoulder at the same time. He remained conscious throughout the event and had full recollection of the fall.

He was examined by the $A \& E$ doctor who found tenderness and limited range of movements in both shoulders. There was no asymmetry of the shoulders. Anteroposterior radiographs of both shoulders (fig $1, \mathrm{~A}$ and $\mathrm{B}$ ) were thought to be normal by the doctor. Consultation was sought with a senior colleague because of the severity of symptoms with seemingly normal radiographs. The senior doctor noted the patient's inability to externally rotate the shoulders which suggested posterior dislocation and so axial views of the shoulders were requested. These clearly showed both shoulders to be dislocated posteriorly (fig 2, A and B). 

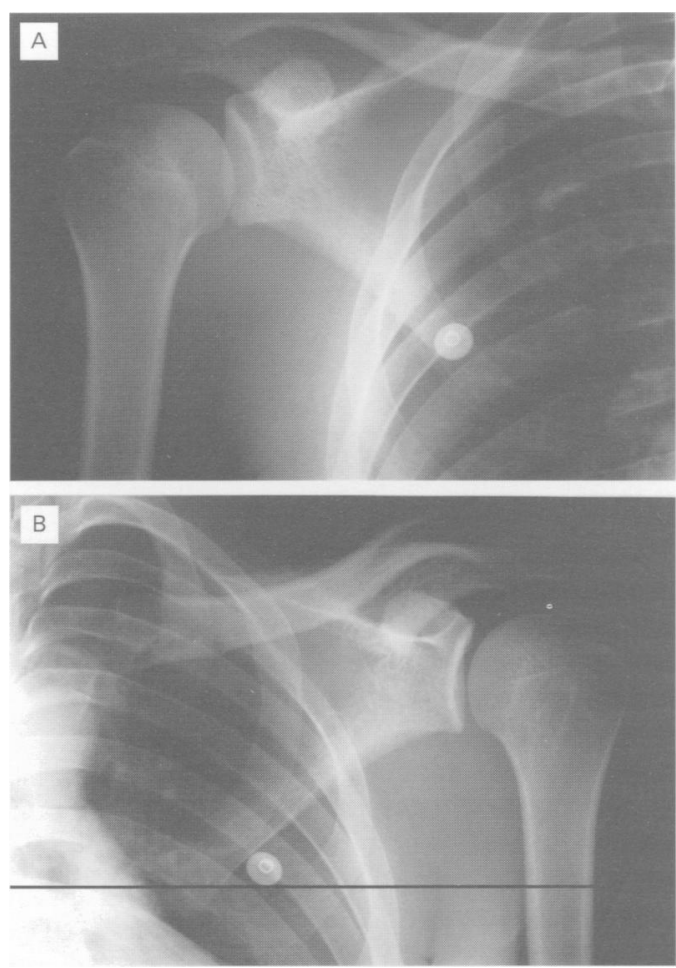

Figure 3 Post-reduction radiographs of right and left shoulders.

The shoulder joint dislocations were reduced without difficulty using intravenous analgesia and sedation. Post-reduction radiographs showed satisfactory positioning of the humeral heads (fig 3, A and B). The shoulders were supported in slings and the patient was referred to the fracture clinic for follow up.

Further inquiry revealed no history of epilepsy or blackouts. There was no history of any orthopaedic or rheumatological problems. Examination showed no signs suggestive of a seizure. In particular he had not been incontinent, his tongue had not been bitten, and there was no sign of a head injury.

\section{Discussion}

Posterior dislocation of the shoulder is an uncommon injury, accounting for between $2 \%$ and $4 \%$ of all shoulder dislocations. It occurs most frequently in patients following convulsions or direct anterior force to the shoulder. ${ }^{1-3}$
In 1952 posterior dislocation of the shoulder was described as often being misdiagnosed, misunderstood, and mistreated. ${ }^{4}$ Cases are still being reported 33 years later. ${ }^{56}$

Several valuable points can help lessen the chance of missing a posterior dislocation. An adequate history and clinical examination should arouse initial suspicion, particularly with the patient's inability to externally rotate the shoulder. Asymmetry of shoulders, often described as an important sign, was not a valuable one in this case, since bilateral dislocation was present and both shoulders were symmetrical. One must be cautious of this sign when there are bilateral symptoms. Likewise it has been recommended that comparison of radiographs of the normal and injured shoulder may be useful, but in this case both sides were the same. This case reinforces the value of obtaining radiographic axial views in cases of trauma to the shoulder area.

Posterior dislocation of the shoulder has been sustained while athletes have been involved in various sports including boxing, wrestling, archery, ${ }^{7}$ and football. We are unaware of any previous report of bilateral posterior dislocation of the shoulder sustained during sporting activity. In fact, we have been unable to find any previous report of bilateral traumatic posterior dislocation of the shoulder which occurred without evidence of an associated convulsion or electrocution.

Doctors and physiotherapists involved in $\mathrm{A} \& \mathrm{E}$, sports medicine, and orthopaedics need to be aware of the pitfalls in diagnosing posterior dislocation of the shoulder and to remember that bilateral posterior dislocation may be present in cases where there is no asymmetry of the shoulders.

1 Karpinski MRK, Porter KM. Bilateral posterior dislocation of the shoulder. Injury 1984;15:274-6.

2 Brown RJ. Bilateral dislocation of the shoulders. Injury 1984;15:267-73.

3 Heller KD, Forst J, Forst R, Cohen B. Posterior dislocation of the shoulder: recommendations for a classification. Arch Orthop Trauma Surg 1994;113:228-31.

4 McLaughlin HL. Posterior dislocation of the shoulder. $f$ Bone foint Surg Am 1952;34:584-90.

5 Elberger ST, Brody G. Bilateral posterior shoulder dislocations. Am $\mathcal{f}$ Emerg Med 1995;13:331-2.

6 Fullerton GM, MacEwan CJ. Bilateral posterior dislocation of the shoulder. Injury 1985;16:428-9.

7 Fukuda H, Neer CS. Archer's shoulder. Recurrent posterior subluxation and dislocation of the shoulder in two archers. Orthopedics 1988;11:171-4. 